\section{Waveband routed optical packet switch: implementa- tion and performance evaluation}

\author{
Christina (Tanya) Politi, ${ }^{a}$ Dimitrios Klonidis, ${ }^{\text {b }}$ \\ Anna Tzanakaki, ${ }^{b}$ Mike O'Mahony, ${ }^{a}$ and \\ loannis Tomkos ${ }^{\text {' }}$ \\ aUniversity of Essex \\ Electronics System Engineering Department \\ CO4 3SQ, Essex, United Kingdom \\ E-mail: cpolit@essex.ac.uk \\ ${ }^{\mathrm{b}}$ Athens Information Technology Center \\ Markopoulo Av. \\ Peania 19002, Athens, Greece
}

\begin{abstract}
An optical packet switch based on wavelength routing and optical waveband conversion is demonstrated. The switch performs header extraction, packet routing, and regeneration and exhibits very good physical performance. (C) 2006 Society of Photo-Optical Instrumentation Engineers.

[DOI: 10.1117/1.2195369]
\end{abstract}

Subject terms: optical communications; optical circuits; switching; optical processing.

Paper 050337 LRR received Apr. 27, 2005; revised manuscript received Jan. 26, 2006; accepted for publication Feb. 8, 2006; published online May 2, 2006.

\section{Introduction}

Optical packet switching (OPS) is seen as a promising solution offering high spectral efficiency through fine switching granularity. ${ }^{1,2}$ Various OPS architectures have been proposed addressing a variety of issues including packet and header format and appropriate switching techniques. ${ }^{1}$ However, a number of issues are still unresolved such as buffering, physical performance, complexity, and cost.

This work examines the performance of an OPS architecture based on waveband converters, a waveband-routed OPS. ${ }^{3}$ A serial header-payload format is utilized with a phase-modulated header and non-return-to-zero amplitude modulated payload. The switching fabric relies on wavelength routing using a set of waveband converters (WBCs) followed by an arrayed waveguide grating (AWG). Waveband routing has been discussed in Ref. 3. WBCs are used to minimize the requirements in terms of wavelength converters, while at the output of the switching subsystem (WBCs and AWG) a single-wavelength conversion (SWC) stage provides flexible wavelength assignment, contention resolution, regeneration, and header deletion. These features significantly reduce the overall component count, cost, and power consumption of a node, supporting reduced capital and operational expenditure in practical networks. ${ }^{3}$

\section{OPS Architecture}

The generic structure of the optical packet switch is illustrated in Fig. 1. Synchronous optical packets of the same length enter the switch through a band demultiplexer. The

0091-3286/2006/\$22.00 @ 2006 SPIE bank of WBCs that follows converts and routes the optical packets to the appropriate output ports through the AWG according to their wavelength. The architecture utilizes grouping of packets in wavebands (group of wavelengths) to enable the use of WBCs; however, this does not impose a banded wavelength channel plan at the transport network level. In particular, although simultaneous switching of all packets at the same waveband is performed, each of the packets is routed individually and independently of the others. The design rules for the application of this scheme are explained in Ref. 3. In the experiment presented here, a packet waveband comprises two packets at different wavelengths. The switch control can be implemented in parallel to the packet routing path, utilizing technology similar to that in Ref. 2. At the input of the switch a 10\% of the signal is tapped for band header decoding (BHD) using differential phase shift keying (DPSK) based decoding through a passive one-bit delayed interferometer (DI). This translates the phase encoded header into the equivalent DPSK intensity variations, which then can be detected and processed by the control. ${ }^{2}$ Header reinsertion (HR) is performed after the SWC by means of a phase modulator.

In this paper a widely tunable four wave mixing (FWM) converter using a semiconductor optical amplifier (SOA) in dual pump configuration ${ }^{2}$ is deployed for the WBC. To enable nonblocking operation of the packet switch, minimizing the number of output wavelength converters, the AWG output ports are combined in a predetermined fashion through passive coupling. ${ }^{3}$ This introduces an increased requirement in AWG dimensions compared to the case with SWC. However, these requirements can be accommodated by existing AWG technology that scales up to $256 \times 256,{ }^{1}$ while multistage configurations can also be utilized. ${ }^{4}$ The output conversion stage operates in tunable-input-fixedoutput mode and is used to assign appropriate wavelengths to the output packets. This assists the contention resolution and minimizes the buffering requirements. In addition to wavelength assignment and contention resolution, the SWC offers regeneration and power equalization, as well as header deletion.

\section{Experimental Configuration}

The experimental configuration shown in Fig. 2 is implemented in order to evaluate the performance of the pro-

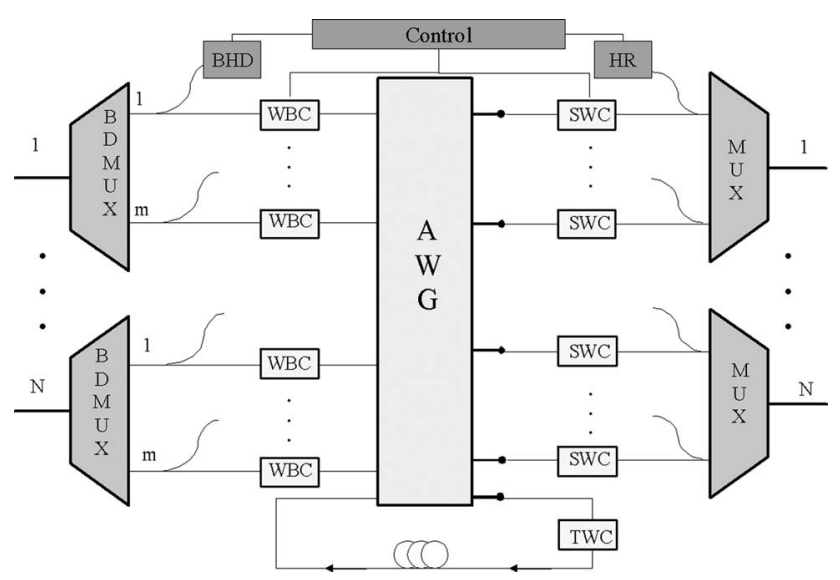

Fig. $1 \mathrm{~N} \times \mathrm{N}$ waveband-based optical packet switch. 


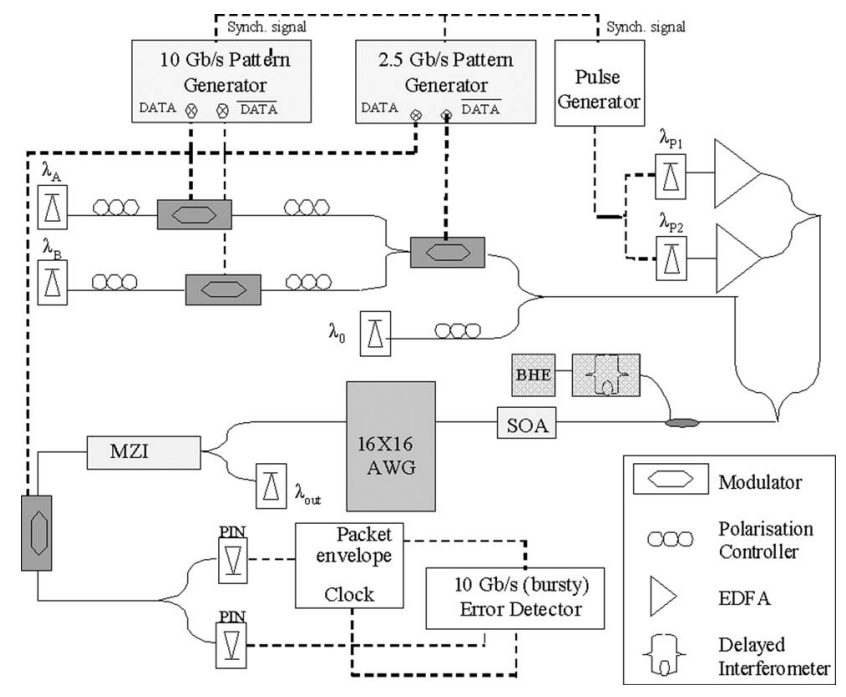

Fig. 2 Experimental setup.

posed OPS design. The OPS demonstrator comprises packet generation, header extraction, waveband conversion, switching, regeneration, and header reinsertion.

Two packet sequences at $\lambda_{\mathrm{A}}=1561.25 \mathrm{~nm}$ and $\lambda_{\mathrm{B}}$ $=1562.05 \mathrm{~nm}$ are modulated at $10 \mathrm{~Gb} / \mathrm{s}$. Each packet consists of 4096 bits. The first 128 bits of the total packet length are set to 'one' (high) and provide a power level on top of which the header bits are encoded on the phase of the carrier at $2.5 \mathrm{~Gb} / \mathrm{s}$. This scheme, previously presented in Ref. 2, offers optimum header encoding, maintaining the extinction ratio of the propagated payload bits at maximum. The guard band between the header and the payload is 12.8 ns in duration, while the guard band between consecutive packets is $72.6 \mathrm{~ns}$. Both packets are modulated with the same header information, and coupled with a fixed pump at $\lambda_{0}=1560.25 \mathrm{~nm}$ and a second wavelength selectable pump. This is obtained through a laser array that selects the appropriate pump required to convert the input waveband packets to the selected output wavelengths. The two laser pumps are at $\lambda_{\mathrm{P} 1}=1547.85 \mathrm{~nm}$ and $\lambda_{\mathrm{P} 2}=1555.3 \mathrm{~nm}$ and are switched appropriately using a pulse generator. They are then introduced into a multi-quantum-well SOA $1 \mathrm{~mm}$ in length, operated at $220 \mathrm{~mA} .^{2}$ This enables the simultaneous conversion of the two input packet streams into different wavebands (on a packet-by-packet basis) and eventually their routing to different outputs of the AWG. As a result, copies of the initial packets at $\lambda_{\mathrm{A}}$ and $\lambda_{\mathrm{B}}$ are produced at $\lambda_{1}=1554.3 \mathrm{~nm}$ and $\lambda_{2}=1553.5 \mathrm{~nm}$ or $\lambda_{3}=1546.85 \mathrm{~nm}$ and $\lambda_{4}=1546.05 \mathrm{~nm}$ depending on the second pump's wavelength $\left(\lambda_{\mathrm{P} 1}\right.$ or $\left.\lambda_{\mathrm{P} 2}\right)$. After routing through the AWG, the packets are converted by a SWC at an output wavelength $\lambda_{\text {out }}=1540.8 \mathrm{~nm}$. The SWC is based on cross-phase modulation $(\mathrm{XPM})$ in a $2 \times 2$ integrated Mach-Zehnder interferometer utilizing SOAs. Each arm consists of two SOAs in a row, each $1 \mathrm{~mm}$ in length. The initial packet sequence at $\lambda_{\mathrm{A}}$ is illustrated in Fig. 3(a). The 1st, 3rd, 4th, and 5th packets of the two input packet sequences at $\lambda_{\mathrm{A}}$ and $\lambda_{\mathrm{B}}$ are converted to $\lambda_{1}$ and $\lambda_{2}$, respectively. The 2 nd packet of the two input packet sequences is converted to $\lambda_{3}$ and $\lambda_{4}$, respectively. The traces of the four converted packets at $\lambda_{1}$, at the output of the AWG, are shown in Fig. 3(b), while the same packets at the output of the XPM converter are illustrated in Fig. 3(c). Similar, Fig. 3(d) and 3(e) illustrate the converted packet at $\lambda_{3}$ and the same one after the XPM converter. Therefore, specific packets are routed to different AWG ports. Additionally, the cross-talk and cross-gain modulation effects, as well as the poor OSNR introduced by the FWM converter, are compensated through the regenerating XPM stage of the SWC at the output. The bit error rate
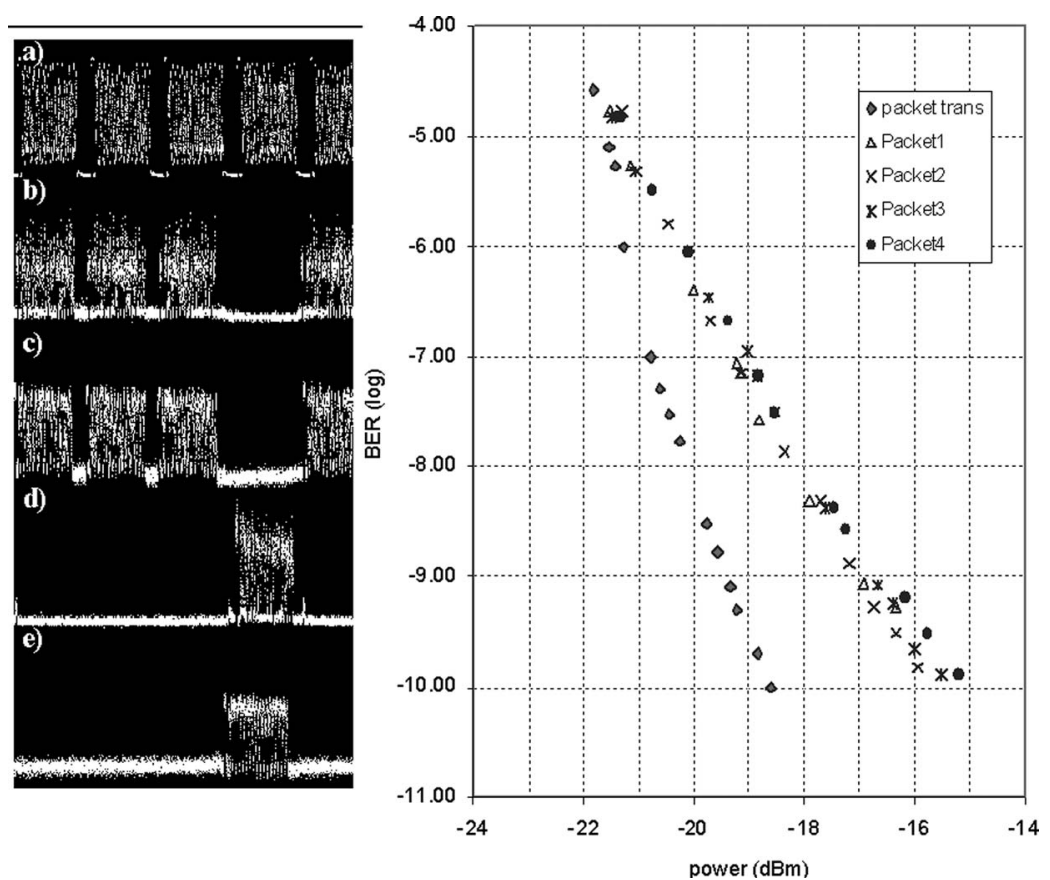

Fig. 3 Packets traces with arbitrary units for comparison and evaluation of the BER measurements as explained in the text. 


\section{OE LETTERS}

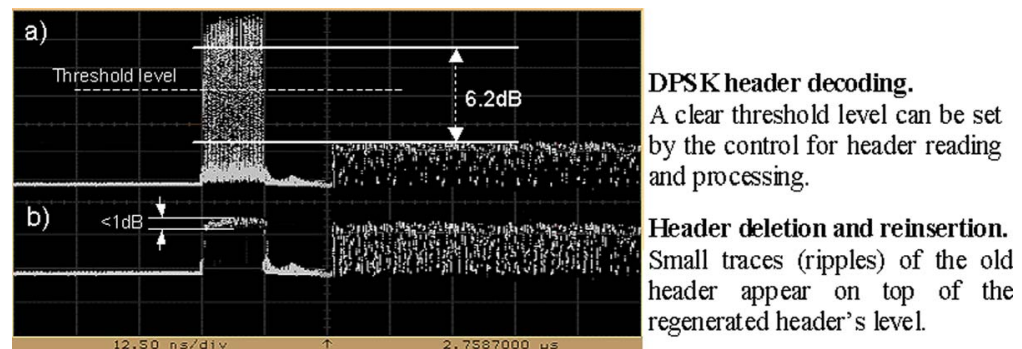

Fig. 4 (a) Decoded header and (b) header's level after XPM SWC.

(BER) curves show that the penalty induced at $10^{-9} \mathrm{BER}$ is $2.6 \mathrm{~dB}$ for packet 1 and increases to $2.9 \mathrm{~dB}$ for packet 4 , due to the wavelength dependent performance of the two converters. The system power penalty was also measured for continuous data operation and was observed to be identical to that of the packet case.

Simultaneous header extraction for both packets with the same characteristics and excellent performance is achieved. An example of the extracted header after the DI is shown in Fig. 4(a). The header payload separation is $6.2 \mathrm{~dB}$ and a clear threshold can be applied by the control. Figure 4(b) shows the header's level after deletion regeneration and reinsertion in phase. Note that small ripples $(<1 \mathrm{~dB})$ appear at the header's level due to the effect of the AWG on the phase-encoded bits and the imperfect regeneration after the XPM processing. However the phase information is fully deleted. These ripples may cause amplitude variations of the DPSK decoded header, especially when packets are routed through a large number of nodes. Phase modulation of the header was arbitrary and no comparative study of the extracted information before and after the reinsertion could be performed due to the differential nature of the coding.

In conclusion, this paper presents a demonstration of an OPS architecture using waveband routing. The switch is based on widely tunable WBCs, an $\mathrm{AWG}$, and regenerative SWCs and minimizes the required component count.

\section{References}

1. S. J. B. Yoo, "Optical packet switching and optical label switching technologies for the next generation optical internet," in Proc. Optical Fibre Comms., Atlanta, Georgia, Paper FS5 (invited) (2003).

2. D. Klonidis, R. Nejabati, C. Politi, M. J. O'Mahony, and D. Simeonidous, "Demonstration of a fully functional and controlled optical packet switch at $40 \mathrm{~Gb} / \mathrm{s}$," in Proc. 30th European Conf. Optical Comm., Stockholm, Sweden, Paper PD Th4.4.5 (Sep. 2004).

3. C. Politi, A. Tzanakaki, D. Klonidis, M. O'Mahony, and I. Tomkos, "Optical cross-connect architecture using waveband conversion and a passive wavelength router," IEE Proc.: Optoelectron. 152(4), 215 221 (August 2005).

4. T. Kawai and H. Obara, "Crosstalk reduction in $\mathrm{N} \times \mathrm{N}$ WDM multi/ demultiplexers by cascading small arrayed waveguide gratings (AWGs)," J. Lightwave Technol. 15(10), 1929-1937 (1997). 